\title{
O FENÔMENO STRONG CROSSOVER NAS GRAMÁTICAS CATEGORIAIS
}

Gelza M. Nunes-Pemberton*

\section{Introdução}

objetivo deste trabalho é apresentar o tratamento que as gramáticas categoriais dão para o fenômeno chamado strong crossover pela gramática gerativa. Na primeira seção apresento as explicações dadas ao strong crossover na teoria da ligação proposta por Chomsky. Na segunda mostro como as gramáticas categoriais trabalham com os fenômenos abarcados pela teoria da ligação. $\mathrm{Na}$ terceira apresento o tratamento que Steedman dá ao strong crossover no quadro das gramáticas categoriais. da FAPESP.

* Aluna do curso de Mestrado em Lingüística da Universidade de São Paulo e bolsista 


\section{O fenômeno strong crossover na gramática gerativa ${ }^{1}$}

A seguinte sentença exibe um fenômeno de agramaticalidade, chamado strong crossover pela gramática gerativa: (1)a. *Who does he think Mary likes $t_{i}$

A sentença (1 a) pode ser interpretada como For which $x$, $x$ thinks Mary likes $x$. Esta mesma interpretação é dada por (1b), versão de (la) sem agramaticalidade.

(1)b. Who does think Mary likes $t_{i}$

Conforme a teoria da ligação, ${ }^{2}$ a configuração da sentença (1a) pode ser descrita como o movimento do elemento-Wh (who) de uma posição- fit $^{3}$ sobre o

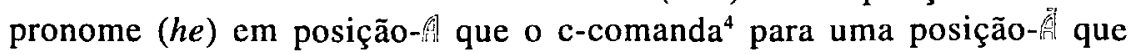
c-comanda o pronome. Como resultado, o elemento- $W h$ liga $^{5}$ o pronome e o pronome liga o vestígio $(t)$ do elemento- $W h .{ }^{6}$ Estando o pronome entre a posi-

1 Conforme Freidin (1994, p. 316), Postal (1971) identificou o strong crossover e Wasow (1979) apresentou a primeira análise teórica do vestígio de strong crossover dentro da teoria da ligação, que mais tarde foi discutida com mais detalhes em Chomsky (1976). Para maiores informações, ver, entre outros, Raposo (1992, p. 239-273) e Freidin (1994, p. 251-305).

2 A teoria da ligação na gramática gerativa trata de condições de relaçāo de antecedência entre posições nas estruturas sintáticas e de condições de interpretação destas relaçōes. As condições sintáticas, chamadas de coindexação, determinam a atribuição ou não-atribuição de um mesmo índice numérico a duas ou mais posições, que podem ser ocupadas por argumentos e operadores. Esclarecese que os índices não têm significados próprios, eles servem unicamente para indicar a relação entre o elemento anafórico e seu antecedente. As condições de ligação estabelecem os traços dos argumentos que ocupam as posições argumentais. Os argumentos podem então ser pronomes (pronomes pessoais), anáforas (os reflexivos -self e os recíprocos each other) e expressões- $\mathrm{R}$ (nomes próprios e expressōes definidas). Esses argumentos são definidos pelas condições $\mathrm{A}, \mathrm{B}$ e $\mathrm{C}$ da teoria de ligação:

(i) Condição A: uma anáfora deve ser ligada na sua categoria de regência.

Condição $B$ : um pronome tem que ser livre na sua categoria de regência.

Condição $C$ : uma expressão referencial (chamada de expressão-R na literatura da teoria da ligaçāo) deve ser localmente A-livre.

3 Raposo (1992, p.131) define a posição-\{̂̉ como posição argumental e a posiçãoposição não argumental. As posições argumentais são ocupadas pelos argumentos da sentença: sujeito, objeto direto e objeto de preposição. Por outro lado, as posições não argumentais compreendem a posição de Comp e posição de adjunção.

4 A c-comanda B se (i) A não domina B e B não domina A e (ii) o primeiro nó que domina A domina também B (Raposo, 1992, p. 32-33).

5 Conforme a teoria da ligação, uma categoria A liga uma categoria B se A é coindexada com B e A c-comanda B. Ser coindexado, por sua vez, significa ter índices referenciais idênticos.

6 Na gramática gerativa, o movimento de uma categoria de uma posição A para uma posição B deixa na posição originária $\mathrm{A}$ uma cópia categorial, sem conteúdo fonético, do constituinte que se moveu. O constituinte movido é chamado antecedente e a cópia é chamada vestígio. 
ção-(1: e a posição-fî̉, pode-se dizer então que o movimento do elemento-Wh cross over o pronome.

Chomsky (1981) considera que a agramaticalidade de (1a) está na relação entre o pronome he e o vestígio $t$, que viola a condição $\mathrm{C}$ da teoria da ligação: uma expressão- $\mathrm{R}^{7}$ (1)-ligada a um pronome. Sendo o vestígio $t$ cópia da categoria que se moveu, uma variável ${ }^{8}$ e uma expressão-R, então $t$ em (la) é uma expressão-R ligada ao pronome he, ou seja, uma variável Aligada; logo (1a) é ilegal.

Uma diferente explicação para o fenômeno do strong crossover dentro da teoria da ligação é dada por Koopman e Sportiche (1983). A partir do algoritmo (2), proposto por Chomsky $(1982$, p.185, 330) para determinar a natureza das categorias vazias, Koopman e Sportiche mostram que o apelo ao princípio C para explicar a agramaticalidade de (1a) parece ser desnecessário.

(2) Sendo $X$ uma categoria em posição-fî,

a. se $X$ é localmente $/$-ligado, $X$ é variável;

b. se $\mathrm{X}$ é livre ou localmente -ligado por $\mathrm{Y}$ com um papel- $\theta$ independente, $\mathrm{X}$ é um pronome.

Segundo Koopman e Sportiche, conforme (2) e conforme a estrutura de strong crossover de (1a) em (3), ${ }^{9}$ o vestígio $t$ é localmente portanto $t$ é um pronome e não uma variável. Por outro lado, he é localmente -ligado por who, portanto é uma variável e não um pronome. Para que a estrutura (1a) seja bem-formada é necessário então que a cadeia wholhe e a categoria vazia $t$ sejam lícitos.

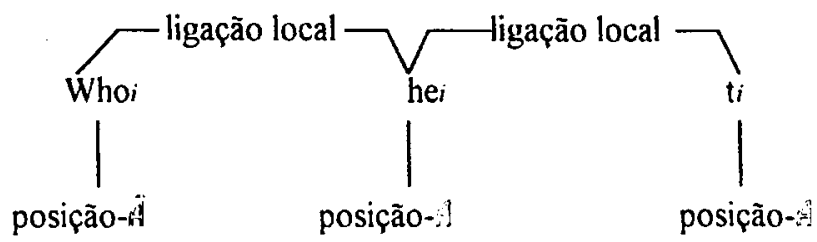

As estruturas (4a) e (5a) de Sportiche (1985, p. 462) mostram que o inglês não permite categorias vazias lexicalizadas. Isso explica a agramaticalidade de (1a) desde que (1a) contém uma variável lexicalizada, que neste caso é he.

7 Conforme a literatura, uma variável é uma expressão- $R$.

8 O vestígio ligado a uma categoria em posição-f̂̉ é chamado de variável. No caso de (1a) então, o vestígio $t$ é uma variável porque estáligado a Who, a uma categoria que está ocupando uma posição-Â.

9 Esta representação é baseada no exemplo (5) de Sportiche (1985, p. 462). 
Assim a cadeia wholhe é mal-formada. Ainda, conforme (4a) e (5a), o inglês não permite que pronomes sejam categorias vazias nestes comportamentos. Logo, (1 a) é agramatical também porque contém um pronome ilícito, um pronome que é uma categoria vazia. Resumindo, na teoria da ligação a configuração de strong crossover é excluída porque o vestígio do elemento wh não corresponde a um tipo de categoria vazia bem-formada. $O$ pronome deve ter

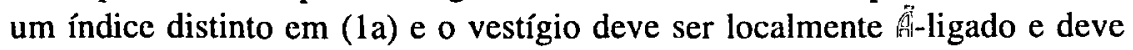
satisfazer a definição de uma variável.

(4)a. *Who, did he see John

b. ${ }^{*} \mathrm{He}_{\mathrm{i}}$ saw $\mathrm{e}_{\mathrm{i}}$

(5)a. *Who did he $e_{i}$ say John slept

b. $* \mathrm{He}_{\mathrm{i}}$ said $\mathrm{e}_{\mathrm{i}}$ left

\section{A teoria da ligação e as gramáticas categoriais}

As gramáticas categoriais são caracterizadas como gramáticas lexicais radicais. Toda a carga sintática e semântica é codificada nas entradas lexicais. Portanto, a descrição do comportamento sintático e semântico de qualquer palavra é dada pela categoria que lhe é atribuída no léxico. Qualquer palavra com comportamentos distintos terá entradas lexicais distintas. O verbo correr, por exemplo, que pode ser tanto transitivo (Maria correu cem metros.) como intransitivo (Maria correu.) terá duas entradas lexicais: (SWN)/N, SW.

As propriedades anafóricas de uma palavra serão então codificadas no léxico. Steedman (1996, p. 17) propõe que as anáforas são sintaticamente idênticas a outros NPs, marcadas com valor + no traço ANA e sua interpretação é uma função própria de reflexivos (self') ou reciprocos (other'):

(6) himself $:=N P_{+A N A, 3 S M}:$ self'

(7) each other $:=N P_{+A N A, P L}$ : other'

Associando os argumentos NP e PP dos verbos com o elemento anafórico que ocorre na posição argumental do verbo, Steedman (1996, p. 17) fornece a regra de reflexivização do verbo transitivo em (8).

(8) $\left(\mathrm{S} \backslash \mathrm{NP}_{a g r}\right) / \mathrm{X}: \mathrm{f} \Rightarrow\left(\mathrm{S} \backslash \mathrm{NP}_{a g r}\right) / \mathrm{X}_{+A N A, a g r}: \lambda g . \lambda y . g f\left(a n a^{\prime} y\right) y$

Em (8) X é NP ou PP e $\Rightarrow$ é um operador relacional de outputs e inputs de regras lexicais. Considerando que a semântica da sentença na abordagem das gramáticas categoriais é construída por combinação de categorias ou apli- 
cação funcional, o elemento anafórico reflete sua ligação no nível de estrutura de argumento-predicado. Sendo assim, sua forma lógica no cálculo $\lambda$ é $g f\left(a n a^{\prime} y\right) y$, onde $g$ é uma variável que realiza a interpretação de anáforas, como self' e other', e ana 'y ${ }^{10}$ é um termo que representa o argumento ligado. Tomando um verbo transitivo como sees (SWP)/NP com um complemento anafórico ligado, a regra (8) nos fornece a categoria lexical (9). ${ }^{11}$ Usando (9) para derivar a sentença Keats sees himself, temos a derivação (10).

(9) sees:= $\left(\mathrm{S} \backslash \mathrm{NP}_{3 S}\right) / \mathrm{NP}_{+A N A, 3 S}: \lambda g . \lambda y \cdot g$ see'(ana'y)y

\begin{tabular}{|c|c|c|}
\hline Keats & sees & himsclf \\
\hline $\begin{array}{l}\overline{N P_{3 S M}} \\
: \text { keats }\end{array}$ & 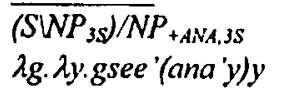 & $\begin{array}{l}\overline{N P_{+A N A, 3 S M A}} \\
: \text { self }\end{array}$ \\
\hline
\end{tabular}

$\mathrm{SWN}_{3 \mathrm{SM}}: \lambda y$. self see $^{\prime}\left(\mathrm{ana}^{\prime} \mathrm{y}\right) \mathrm{y}$

S : self'see'(ana'keats')keats'

Os verbos como talk, embora transitivos como see, têm entrada lexical distinta porque têm regência diferente da de see. Steedman (1996, p. 18) fornece a seguinte regra de reflexivização para verbos como talk:

(11) $P P_{\text {To,ana,agr }} / N P_{\text {anu,agr }}: \lambda g . g$

A aplicação da regra (11) à forma verbal talks produz a categoria (12), que por sua vez fornece a derivação da sentença Keats talks to himself em (13). (12) talks := $\left(S W P_{3 S}\right) / P P_{\text {To. }+ \text { ANA.JS' }}: \lambda g . y . g$ talkto'(ana'y)y

10 O termo ana'y (nos exemplos, ana'keats'), que traduz a anáfora na estrutura de argumento-predicado, é tratado como um pro-termo.

11 Exemplo (23) de Steedman (1996, p. 17). 
Keats

$N I_{3 \times 44}$

:keats talks

$\left(S: N P_{3,}\right) P P(1), A N A, 35$

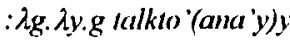

to

himself

PP TO.anu.ugr $N P_{\text {ana.ugr }}$

: $\lambda g . g$
$N P+A N A, 3$ W

$\therefore s e l f$

$$
\text { PPT. ANASW : self' }
$$

$S i N P_{3 s}:$ Ay.self'talkto' (ama' $\left.y\right)_{y}$

S : .se'f' 'ralkto '(ana 'keats ')ke'ats'

Steedman (1996, p. 15) defende que estruturas como self'see'(ana'keats') keats' são equivalentes a árvores binárias, como (14), e prescrvam as noções de dominância e comando dessas árvores.

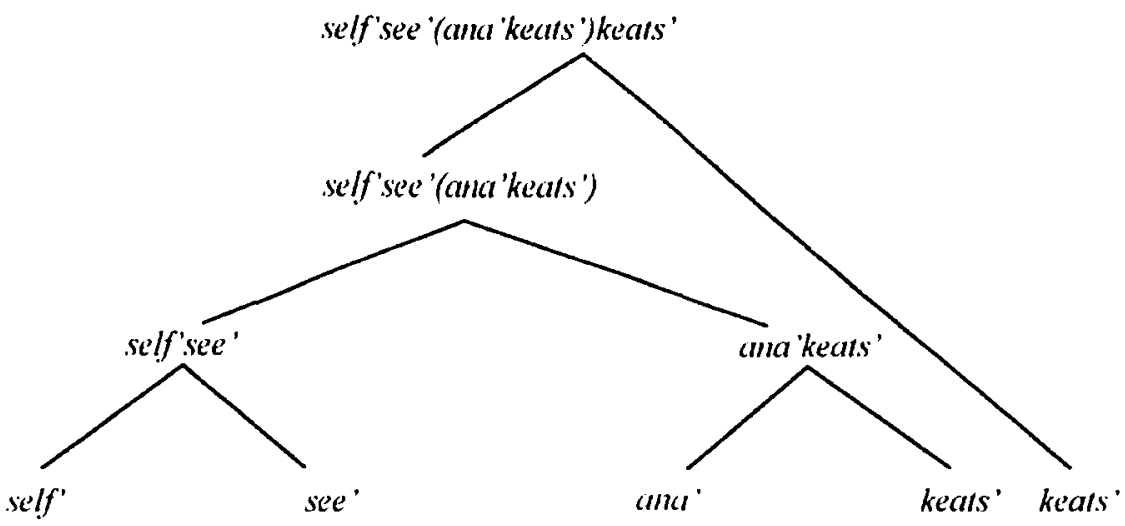

A partir daí, Steedman (1996) incorpora a teoria da ligação e do controle, definida por Bach e Partee (1980) em termos de forma lógica, à gramática categorial original de Adjukiewicz e Bar-Hillel.

As relações de c-comando e ligação são definidas em Steedman (1996, p. 19) como (15) e (16), respectivamente. 


\section{(15) C-comando}

Um nó $\alpha$ na estrutura de argumento-predicado c-comanda um nó $\beta$, se o nó que imediatamente domina $\alpha$ domina também $\beta$ e $\alpha$ não domina $\beta$; ou, se $\alpha$ é $o$ argumento no pro-termo e o pro-termo c-comanda $\beta$.

(16) Ligação

Um nó numa estrutura de argumento-predicado é ligado quando é idêntico ${ }^{12}$ ao nó que o c-comanda na estrutura de argumento-predicado.

Para compreender melhor estas relações, eu as ilustro em (17).

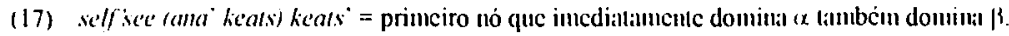

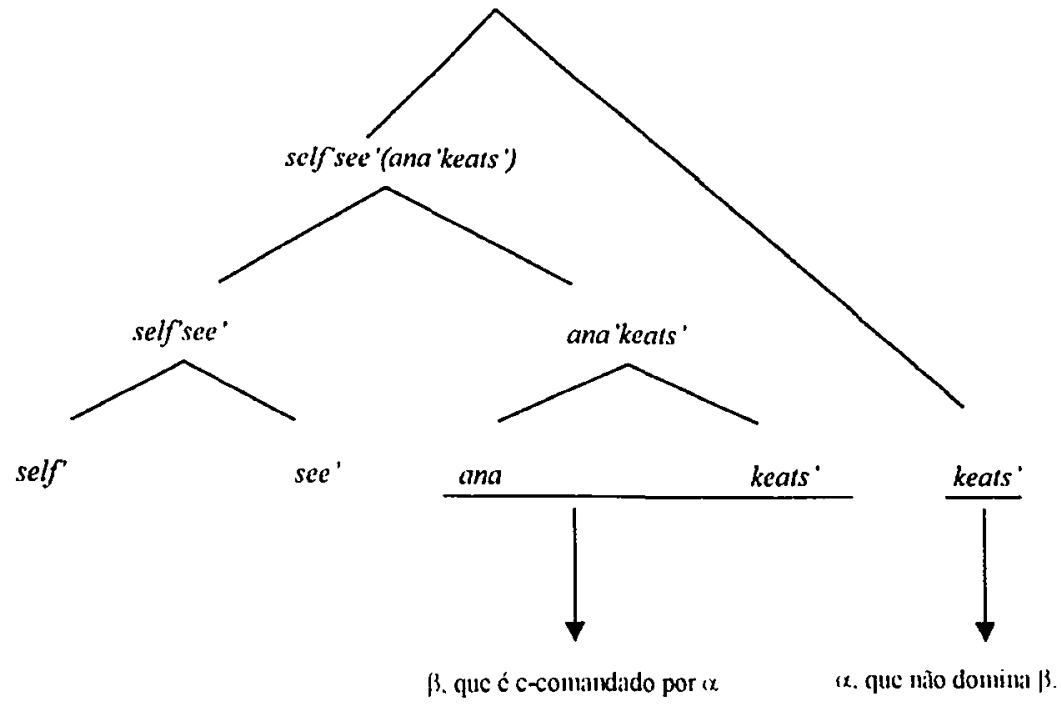

O tratamento lexical de anáfora ligada define então seu domínio de localidade como domínio da sua categoria verbal lexical. Dessa forma, as condições A e B da teoria da ligação na gramática gerativa são realizadas nas gramáticas categoriais via verbos reflexivizados. Para alcançar a explicação de fatos como a não-ocorrência de sujeitos anafóricos ligados (cf. (19)), Steedman (1996, p. 20) propõe a condição C:

${ }^{12} \mathrm{O}$ autor deixa claro que idêntico aqui refere a identidade literal, ou seja, dois nós produzem a unificação de duas ocorrências de mesma variável com o mesmo conteúdo. Ex.: (ana'y')y ou (ana'keats')keats'. 
(18) Condição C

Somente o argumento pro-termo pode ser ligado.

(19)a. *Each other saw the dogs.

b. *other'see'dogs'(ana'dogs')

Desde que somente um pro-termo pode ser ligado e dogs' não é um protermo, (19) é agramatical porque dogs' é ligado. A estrutura arbórea em (20) do exemplo (19) mostra este fato com mais clareza.

(20)

*other 'see 'dogs (ana'dogs')

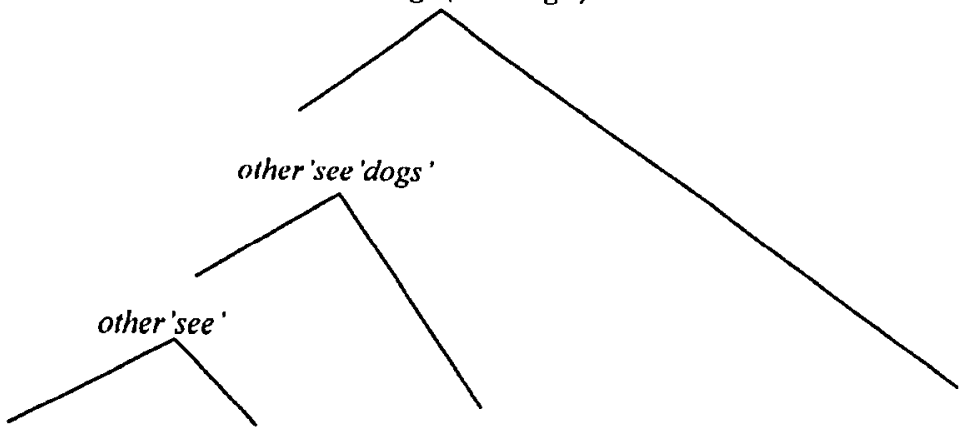

other'

see $e^{\circ}$

dogs

(ana'dogs')

A condição C captura também a assimetria de (22). Dada a entrada lexical do pronome em (21), podemos ver que o pronome he em (22a) tem um pro-termo ligado lexicalmente, ou seja, tem um pro-termo que é c-comandado pela variável $x$. A representação arbórea de (22a) em (23a) esclarece esse fato. A sentença (22b) por sua vez é agramatical, pois, como nos mostra (23b), o elemento ligado every man não é um pro-termo.

(21) him := NPpron :pro' $x$

(22)a. Every man thinks that he is a genius.

thinks' (is'a genius'(pro'y)) $x$

b. * $\mathrm{He}_{\mathrm{i}}$ thinks that every man $_{\mathrm{i}}$ is a genius.

thinks'(is'a genius' $x$ )(pro'y) 


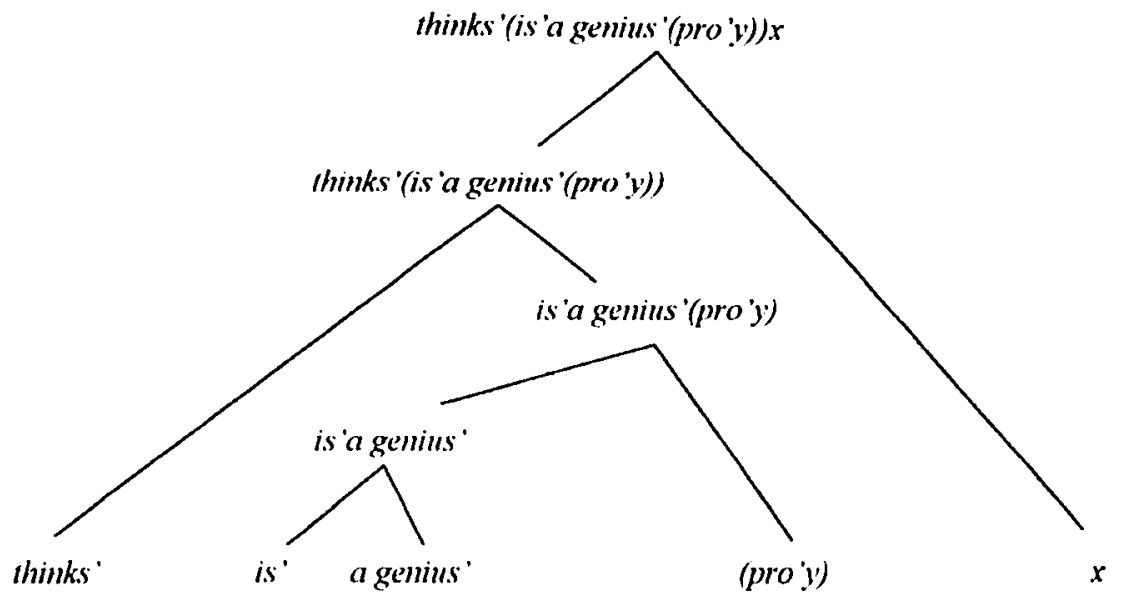

(23b)

thinks '(is 'a genins 'x) (pro'y)

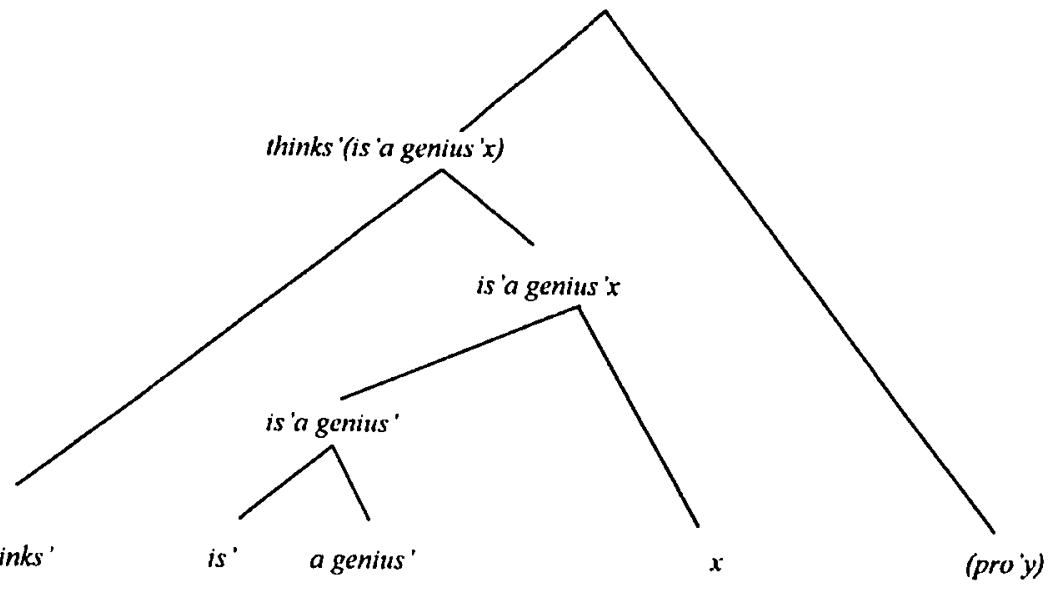

A condição $C$ explica também a agramaticalidade de sentenças em que um pronome não-oblíquo c-comanda um argumento oblíquo extraído (cf. (24)) o "pied-piping" em (25) onde Whose pictures traduz-se como pictures' (of' $x$ ). A condição $C$ exclui as sentenças (24a) e (25a) porque nelas o pronome ligado unifica $x$ a $y$, além de ser ligado a um pro-termo, ou seja, ser c-comandado por um pro-termo.

(24)a. *a man Who(m)i I told him $i$ that Mary liked

b. a man Who(m) $i$ I told that Mary liked himi 
(25)a. * a man pictures of Who(m) $i$ he $i$ (thinks that Mary) likes

b. *a man Whose $i$ pictures he $i$ (thinks that Mary) likes $S / N P$ : $\lambda x$. thinks'(like' (pictures'(of'x)) mary') (pro'y)

\section{O tratamento do fenômeno strong crossover pelas gramáticas categoriais}

A agramaticalidade de sentenças com fenômenos tratados como strong crossover pela gramática gerativa é resolvida no léxico, ou seja, é explicada por mecanismos categoriais: regra de reflexivização e violação da condição $\mathrm{C}$ de Steedman (1996).

A sentença (26), por exemplo, exibe o fenômeno identificado como strong crossover pela gramática gerativa.

(26) *a man Who(m)i hei thinks that Mary likes

Aplicando a regra de reflexivização em (26), obtemos a estrutura de argumento-predicado em (27) e a estrutura arbórea em (28). Podemos ver então que há uma variável $y$ ligada a uma variável $x$ e que a variável $y$ é um pronome com um pro-termo ligado, um pronome (pro'y) que c-comanda $x$. Em outras palavras, em (26) temos um elemento ligado $(x)$ e esse elemento não é um pro-termo. Logo, temos violação da condição $C$.

(27) $S / N P$ : $\lambda x$. thinks'(like'x' mary') (pro'y)

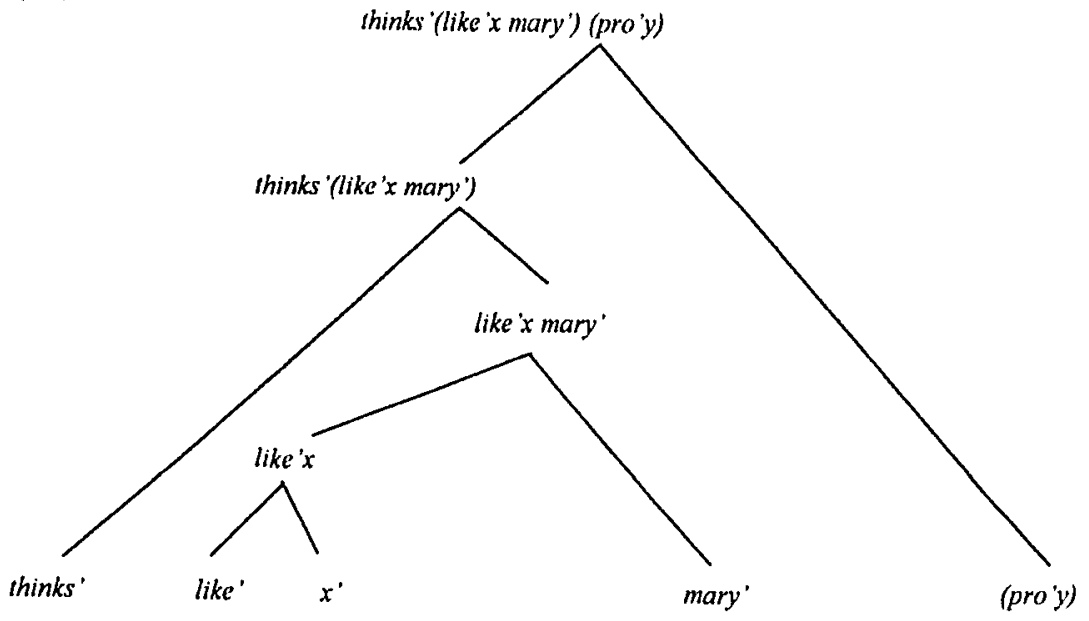


Por outro lado, como podemos ver em (29b) e (30), o único elemento ligado, o pro'y, é um pro-termo. Portanto, a sentença (29) é boa.

(29)a. a man Whoi thinks that Mary likes himi

b. SWP : $\lambda x$. thinks'(like'(pro'y) mary') $x$

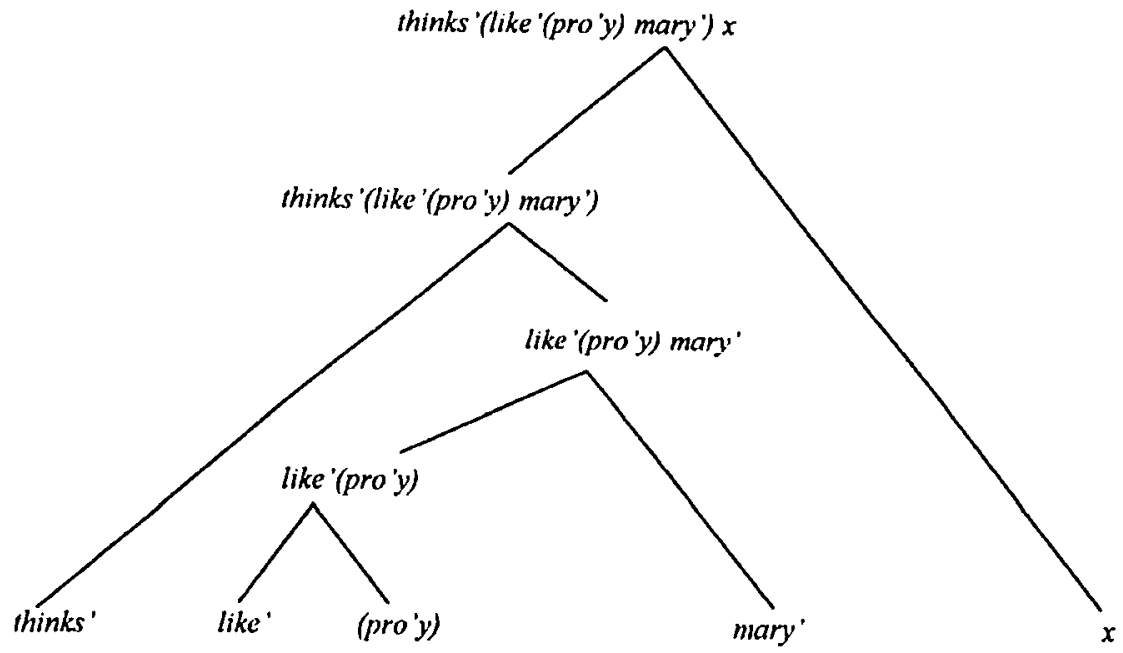

\section{Conclusão}

Para as gramáticas de estruturas frasais, a agramaticalidade de sentenças com strong crossover é resultado de violação de regra de estrutura frasal. No caso da gramática gerativa, tal agramaticalidade é explicada pela violação da condição $\mathrm{C}$ da teoria da ligação. No entanto, para as gramáticas lexicalistas, a rigor, o strong crossover não existe. Nas gramáticas categoriais, a agramaticalidade de exemplos como (26) é explicada por mecanismos de outra natureza.

Observa-se então uma certa equivalência "fraca" entre os dois tipos de gramática: ambas são capazes de explicar adequadamente os fenômenos gramaticais e agramaticais da língua, embora lancem mão de mecanismos de naturezas completamente distintas. Enquanto as gramáticas estruturais utilizam princípios de estrutura frasal para explicar os fenômenos de agramaticalidade, as gramáticas lexicais usam as entradas lexicais. 


\section{RESUMO}

O objetivo deste trabalho é apresentar o tratamento que as gramáticas categoriais dão para o fenômeno chamado strong crossover pela gramática gerativa. Para isso, apresento as explicações dadas ao strong crossover na teoria da ligação proposta por Chomsky e mostro como as gramáticas categoriais trabalham com os fenômenos abarcados pela teoria da ligação.

Palavras-chave: sintaxe, gramática categorial, strong crossover.

\section{ABSTRACT}

This paper presents the analysis which categorial grammars give to the phenomenon called strong crossover by generative grammar. For this, I of fer the analysis of strong crossover in binding theory proposed by Chomsky, and show how the categorial grammars deal with the phenomena included in binding theory.

Key-words: syntax, categorial grammar, strong crossover.

\section{REFERÊNCIAS BIBLIOGRÁFICAS}

BACH, E.; PARTEE, B. Anaphora and semantic structure. In: CLS 16: Papers from the parasession on pronouns and anaphora. Chicago: Chicago Linguistics Society, University of Chicago, 1980. p. 1-28.

CHOMSKY, N. Conditions on rules of grammar. Linguistic Analysis, v. 2, p. 303-351, 1976.

Lectures on government and binding. Foris: Dordrecht, 1981.

KOOPMAN, H.; SPORTICHE, D. Variables and the bijection principle. The Linguistic Review, v. 2, p. 139-160, 1983.

RAPOSO, E. Teoria da gramática: a faculdade da linguagem. Lisboa: Caminho, 1992. FREIDIN, R. Foundations of generative syntax. Cambridge, Massachusetts: The MIT Press, 1994.

POSTAL, P. Crossover phenomena. New York: Holt, Rinehart and Winston, 1971.

SPORTICHE, D. Remarks on crossover. Linguistic Inquiry, v. 16, n. 3, p. 460-469, 1985.

STEEDMAN, M. Surface structure and interpretation. Cambridge, Massachusetts: The MIT Press, 1996.

WASOW, T. Anaphora in generative grammar. Ghent: E. Story-Scientia, 1979.

WOOD, M. McGee. Categorial grammars. London: Routledge, 1993. 